\title{
Monte Carlo simulations of $\mathrm{NiFe}_{2} \mathrm{O}_{4}$ Nanoparticles
}

\author{
Chenggang Zhou, ${ }^{1,2}$ T. C. Schulthess, ${ }^{1}$ and D. P. Landau ${ }^{2}$ \\ ${ }^{1}$ Computer Science and Mathematics Division, \\ Oak Ridge National Laboratory, P. O. Box 2008, \\ MS6493, Oak Ridge Tennessee, 37831-6493 USA \\ ${ }^{2}$ Center for simulational physics, University of Georgia, Athens Georgia, 30602 USA
}

(Dated: November 6, 2018)

\begin{abstract}
We use Monte Carlo simulations to study $\mathrm{NiFe}_{2} \mathrm{O}_{4}$ nanoparticles. Finite size and surface effects differentiate them from their bulk counterparts. A continuous version of the Wang-Landau algorithm is used to calculate the joint density of states $g\left(M_{z}, E\right)$ efficiently. From $g\left(M_{z}, E\right)$, we obtain the Bragg-Williams free energy of the particle, and other physical quantities. The hysteresis is observed when the nanoparticles have both surface disorder and surface anisotropy. We found that the finite coercivity is the result of interplay between surface disorder and surface anisotropy. If the surface disorder is absent or the surface anisotropy is relatively weak, the nanoparticles often exhibit superparamagnetism.

PACS numbers: 75.50.-y 75.60.-d 75.75.+a
\end{abstract}




\section{INTRODUCTION}

Many magnetic nanoparticles are found to be superparamagnets, $\frac{1}{1}$ since they are not sufficiently big to contain more than one magnetic domain. On the other hand, a large fraction of the spins are located near the surface of the nanoparticle, and their local environments have lower symmetries than the bulk. These spins may prefer to align to directions different from the bulk ordering direction, thus reducing the total magnetization. This surface anisotropy might contribute significantly to the total magnetoanisotropy and the hysteresis

of the nanoparticle, ${ }^{2}$ e.g. ferrimagnetic $\mathrm{NiFe}_{2} \mathrm{O}_{4}$ nanoparticles. The bulk $\mathrm{NiFe}_{2} \mathrm{O}_{4}$ is a ferrimagnet with a Neel temperature $T_{N}=838 \mathrm{~K}$. The ball-milled nanoparticles have an average radius of $6.5 \mathrm{~nm}^{3}$ and have been previously found to show a slightly open hysteresis loop at low temperatures. $\frac{4,5}{2}$

In this paper, we use the heatbath algorithm and the Wang-Landau algorithm to calculate the finite-temperature properties of individual $\mathrm{NiFe}_{2} \mathrm{O}_{4}$ nanoparticles. The heatbath algorithm ${ }^{6.7}$ runs at a certain temperature and external magnetic field. However, the heatbath algorithm samples does not directly generate the hysteresis, because the canonical ensemble it samples is dominated by the ground state rather than a meta-stable state, which contributes to the hysteresis. We use a continuous version of the Wang-Landau algorithm ${ }^{10}$ to calculate the joint density of states of the nanoparticles, $g\left(M_{z}, E\right)$, where $M$ and $E$ are magnetization and internal energy of the nanoparticle respectively. $g\left(M_{z}, E\right)$ contains complete information of the nanoparticle at any temperature and in any magnetic field.

\section{MODEL OF $\mathrm{NiFe}_{2} \mathrm{O}_{4}$ NANOPARTICLES AND COMPUTATIONAL METHODS}

Bulk $\mathrm{NiFe}_{2} \mathrm{O}_{4}$ has an inverted spinel structure of lattice constant $8.34 \AA$. The eight tetrahedral sites in one unit cell are occupied by $\mathrm{Fe}^{3+}$, while eight $\mathrm{Fe}^{3+}$ and eight $\mathrm{Ni}^{2+}$ are distributed on the sixteen octahedral sites. We assume the ions on the octahedral sites have Verwey order, i.e. the $\mathrm{Fe}^{3+}$ and $\mathrm{Ni}^{2+}$ occupy (100) planes alternatively. In this system, $S=5 / 2$ for $\mathrm{Fe}^{3+}$, and $S=1$ for $\mathrm{Ni}^{2+}$. The Hamiltonian for the magnetic structure of the bulk consists of Heisenberg indirect exchange:

$$
\mathcal{H}_{\text {bulk }}=-\sum_{<i, j>} J_{i j} \mathbf{S}_{i} \cdot \mathbf{S}_{j}-\mathbf{h} \cdot \sum_{i} \mathbf{S}_{\mathbf{i}}
$$


where the summation is over nearest and next nearest pairs with nonzero exchange $J_{i j}$. The exchange constants $J_{i j}$ are taken from Ref. [4] and we use semiclassical approximation for the spins. In the ground state, $\mathrm{Fe}^{3+}$ spins on tetrahedral sites are antiparallel to $\mathrm{Fe}^{3+}$ spins on octahedral sites, while $\mathrm{Ni}^{2+}$ spins align with $\mathrm{Fe}^{3+}$ spins on octahedral sites. The cubic anisotropy $\left(K_{1}=-8.7 \times 10^{4} \mathrm{erg} / \mathrm{cm}^{3}\right.$ at $\left.77 \mathrm{~K}^{11}\right)$ is negligible for these nanoparticles.

We model the nanoparticles by cutting off those spins outside a sphere of a given radius. The center of the sphere is randomly selected, which might not be a lattice point. Following the previous work ${ }^{4}$, we randomly remove a small fraction of surface spins to model surface disorder, and use uniaxial anisotropy for the surface spins:

$$
\mathcal{H}_{\mathrm{A}}=-k_{s} \sum\left(\hat{\mathbf{s}}_{i} \cdot \hat{\mathbf{n}}_{i}\right)^{2}
$$

where the unit vector $\hat{\mathbf{s}}_{i}$ points in the direction of spin $\mathbf{S}_{i}$, and the unit vector $\hat{\mathbf{n}}_{i}$ is chosen to be parallel to $\sum_{j}^{n n}\left(\mathbf{r}_{i}-\mathbf{r}_{j}\right)$ where $\mathbf{r}_{j}$ are positions of nearest neighbors of $\mathbf{r}_{i}$ in the nanoparticle. As a result, the surface anisotropy term vanishes if local inversion symmetry exists for that spin. $k_{s}$ in Eq. (2) characterizes the strength of the anisotropy. A realistic model would have $k_{s}$ dependent on the local environment of the spin. but in our simulations, $k_{s}$ is a positive tunable constant. We use $0,2.5 \mathrm{~K}, 300 \mathrm{~K}$, and $500 \mathrm{~K}$ for $k_{s}$, to observe the effect of different strength of the anisotropy.

We use the heatbath algorithm ${ }^{6.7}$ and the improved Wang-Landau algorithm 9.10 in our simulations. The heatbath algorithm reaches equilibrium in presence of disorder and anisotropy much faster than the Metropolis algorithm. $\stackrel{8}{*}$ The continuous version of the WangLandau algorithm ${ }^{10}$ calculates the joint density of states

$$
g\left(M_{z}, E\right)=\int \delta\left[\frac{\mathcal{H}(\mathbf{S})}{N}-E\right] \delta\left(\frac{\sum_{i} \mathbf{S}_{i}^{z}}{N}-M_{z}\right) d \mu(\mathbf{S}),
$$

where $N$ is the number of spins, $\mathbf{S}$ collectively denotes all the spins in the model, $d \mu(\mathbf{S})=$ $\prod_{i} S_{i}^{-2} \delta\left(S_{i}^{2}-\mathbf{S}_{i}^{2}\right) d S_{i}^{x} d S_{i}^{y} d S_{i}^{z}$ is the volume measure of the phase space, $E$ and $M_{z}$ are the average energy per spin and average magnetization per spin. With $g\left(M_{z}, E\right)$ in Eq. (3) at hand, one calculate the partition function, and the Bragg-Williams free energy defined as a function of temperature and magnetization: $F\left(M_{z}, T\right)=-k_{B} T \ln \int g\left(M_{z}, E\right) e^{-\beta E N} d E$. If the system exhibits a first order phase transition, $F\left(M_{z}, T\right)$ has double minima at constant $T$. The free energy difference between the ground state and the metastable state as well as the free energy barrier between them are obtained from $F\left(M_{z}, T\right)$. 


\section{RESULTS AND DISCUSSION}

Using the heatbath algorithm, we calculated the specific heat and the Binder cumulant of magnetization for bulk samples of different sizes with periodic boundary condition. With finite size scaling, we obtain the Neel temperature for ferrimagnetic phase $T_{N}=869 \mathrm{~K}$, which is about $4 \%$ higher than the experimental value. We also compared the simulations for bulk material to those for the nanoparticles. Figure 1 shows the effect of the size and surface of the nanoparticle.

The peak in the specific heat of the nanoparticle appears at a lower temperature, about 2(
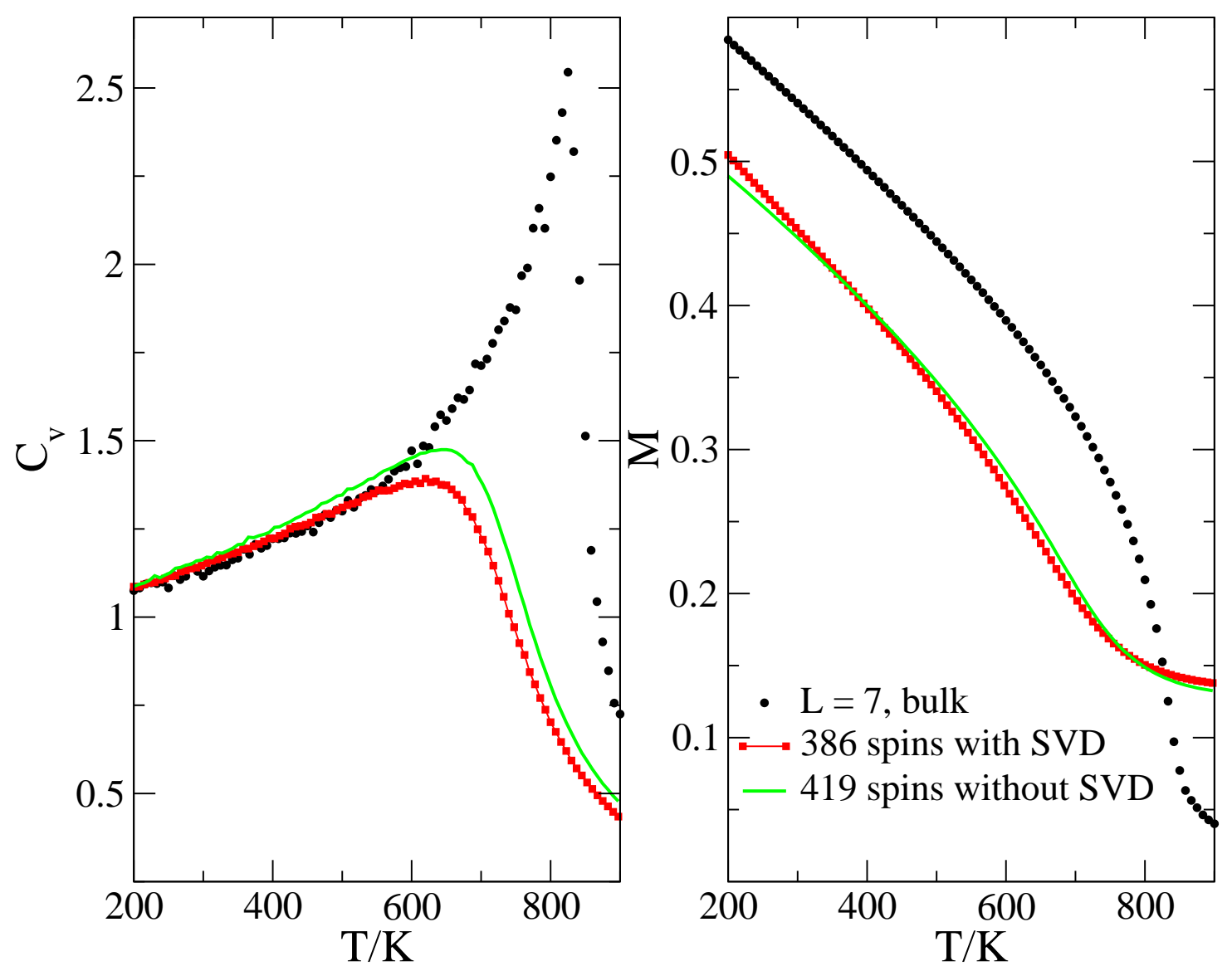

FIG. 1: Comparison between the bulk material and the nanoparticle. Left: the specific heat of nanoparticles has a smooth peak below the Neel temperature of the bulk material. Right: the reduced magnetization of the nanoparticle. The bulk sample is a cubic with 7 unit cells along each axis. 
as $M=N^{-1}\left|\sum_{i=1}^{N} \mathbf{S}_{i}\right|$. The magnetization of the nanoparticle is substantially suppressed. Near the transition temperature, the magnetization of the nanoparticle gradually ramps up with decreasing temperature, while the bulk materials show a magnetization curve typical of the ferrimagnetic phase transition.

Figure 2 shows $g\left(M_{z}, E\right)$ for a typical nanoparticle with surface disorder and 387 spins. Figure 2(a) shows $g\left(M_{z}, E\right)$ for a large energy range: $-1200 \mathrm{~K}<E<0 \mathrm{~K}$, which illustrates the generic behavior of density of states of these nanoparticles. Here the magnetization $M_{z}$ is the average $z$-component of the spins. At high energies, $g\left(M_{z}, E\right)$ has a single maximum value at $M_{z}=0$ for constant $E$. At low energies, this single maximum is replaced by a relatively flat part, i.e. $\partial g\left(M_{z}, E\right) / \partial M_{z} \approx 0$, which indicates a non-zero spontaneous magnetization. The energy range of Fig. 2(a) is sufficient for calculating temperature dependent properties for approximately $T>400 \mathrm{~K}$. The magnetization as a function of temperature and external field is shown in Fig. 2(b). A spontaneous magnetization develops at about $T=700 \mathrm{~K}$, consistent with the reduced transition temperature shown in Fig. 1. Since the model has continuous degrees of freedom, $g\left(M_{z}, E\right)$ is logarithmically divergent near the ground state energy ${ }^{10}$ Thus, the lowest energy that a simulation can efficiently sample is restricted by its resolution in energy. Fig. 2(c) and (d) show $g\left(M_{z}, E\right)$ at low energies calculated with higher resolution in energy. Their energy ranges allow a temperature range $180 \mathrm{~K}<T<220 \mathrm{~K}$ when the $g\left(M_{z}, E\right)$ is used to calculate temperature dependent quantities. In Fig. 2(c), a double-valley structure appears between $M=-0.4$ and $M=0.4$. This structure is responsible for a finite coercivity, because the magnetization is blocked by it when the magnetic field is slightly reversed. The double valley structure becomes more pronounced at lower temperatures. On the contrary, in Fig. 2(d), the density of states of the same nanoparticle with a reduced surface anisotropy constant $k_{s}=2.5 \mathrm{~K}$, does not show any noticeable structure as in Fig. 2(c). The energy range in (d) is higher than that in (c) because of an over all energy shift caused by the change in surface anisotropy. However the energy range of (d) corresponds to a temperature range of $40 \mathrm{~K}<T<80 \mathrm{~K}$.

The lack of a finite coercivity in Fig. 3) (d) is due to the result of the rotational symmetry. Obviously the surface disorder preserves the rotational symmetry. Although there is a small $k_{s}$, the surface anisotropy is too weak to destroy the rotational symmetry at temperatures much larger than $k_{s}$. Consequently, the nanoparticle is well described by superparamagnetism. 
When we assume a strong surface anisotropy, which is "larger" than the thermal energy $k T$ and comparable to the exchange field on the surface, then we indeed observe various structures in $g\left(M_{z}, E\right)$, which are responsible for coercivity at finite temperatures. Figure 3 shows four examples of this behavior. We plot the Bragg-Williams free energy per spin $F\left(M_{z}, T\right) / N$ in Fig. 3. Clearly, all of these four examples appear to have a barrier of free energy between two states of spontaneous magnetization, also small oscillations on top of the barrier is shown in Fig. 3 (b).

To observe the effect of the intermediate surface anisotropy, we used $k_{s}=50 \mathrm{~K}$ and performed the calculation with the same set of nanoparticles that we used for the weak and strong surface anisotropy. We found most of them do not appear to have a noticeable depression in the density of states or a peak the free energy even at low temperatures with a few exceptions. One of them is the nanoparticle used to do the calculation for Fig. 3(d), it
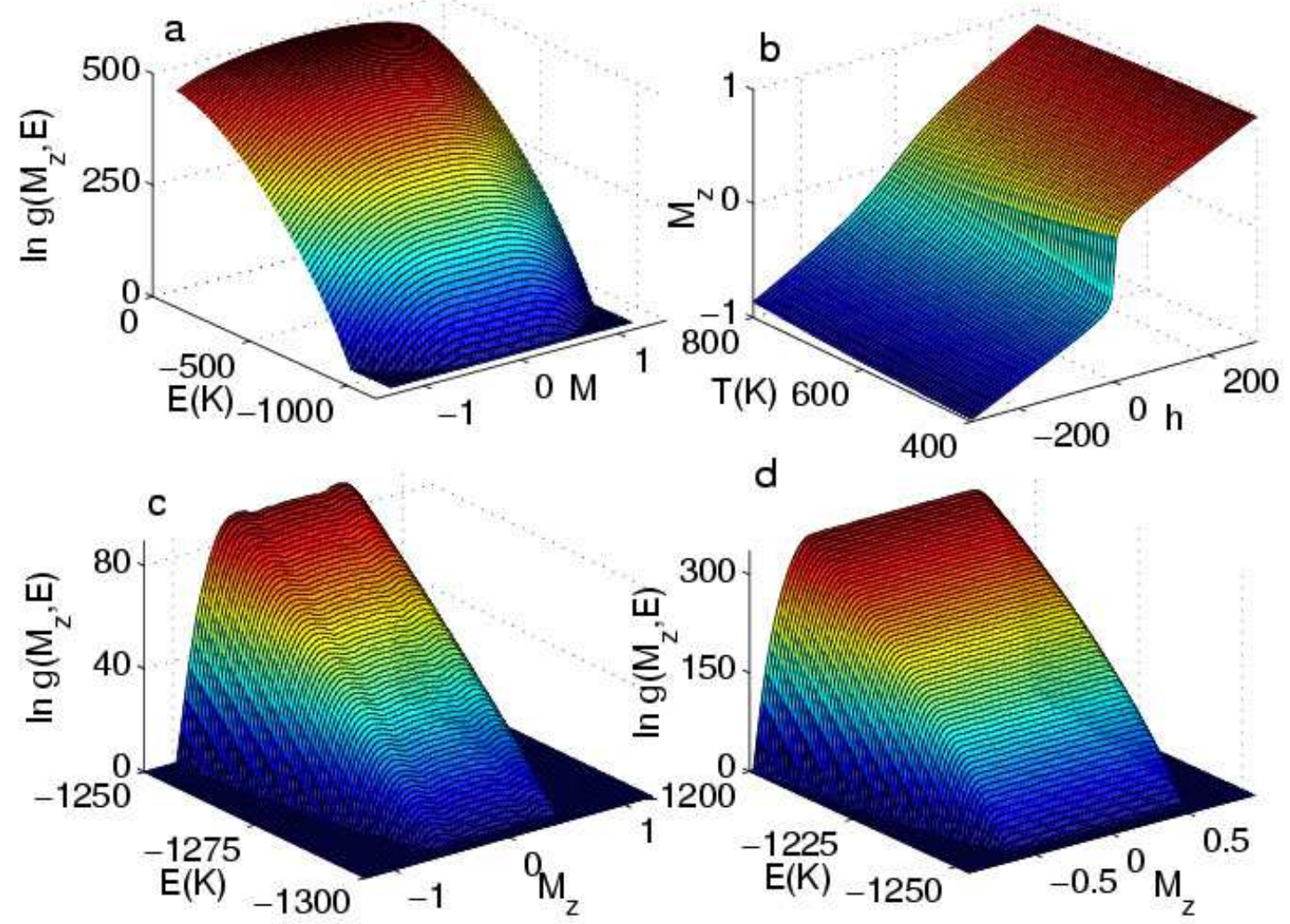

FIG. 2: Density of states and magnetization of a typical nanoparticle containing 387 spins. The normalization constant in the density of states is arbitrarily selected. See the text for detailed explanations. 

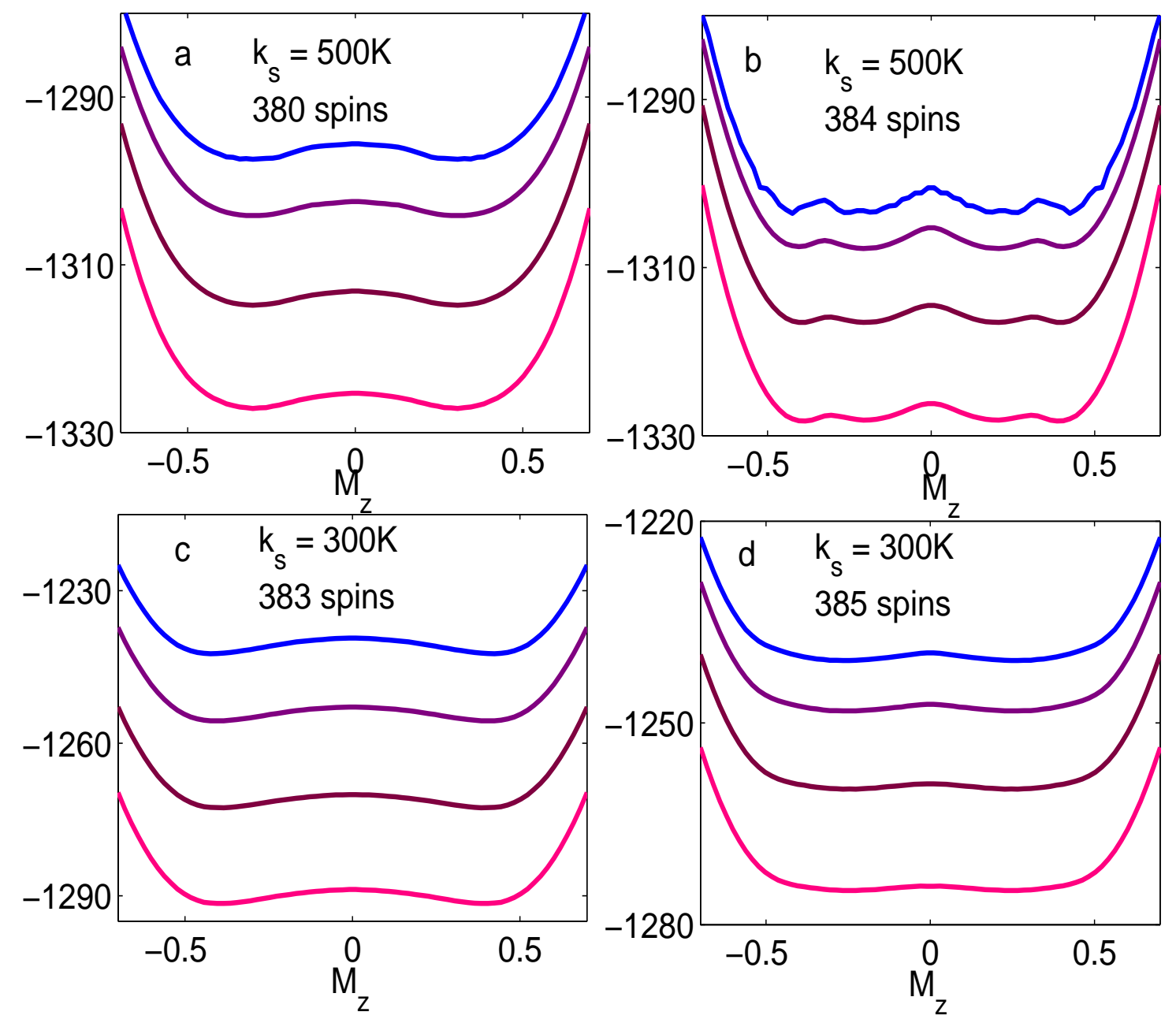

FIG. 3: Bragg-Williams free energy per spin, a variety of structures are observed in nanoparticles with strong surface anisotropy and disorder, at relatively high temperature. In each panel, four free energy curves are for temperatures $187 \mathrm{~K}, 216 \mathrm{~K}, 244 \mathrm{~K}$, and $272 \mathrm{~K}$ respectively from top to bottom.

was found that with $k_{s}=50 \mathrm{~K}$, its Bragg-Williams free energy for $35 \mathrm{~K}<T<55 \mathrm{~K}$ appears to have the similar shape as shown in Fig 3(d).

In summary, we have studied the magnetic properties of $\mathrm{NiFe}_{2} \mathrm{O}_{4}$ nanoparticles using two kinds of Monte Carlo simulations. We have found that only both surface disorder and a pretty strong surface anisotropy are required to develop the hysteresis. We have also noticed that a strong surface anisotropy is required because our model has a near-spherical shape. The surface anisotropies at different positions are likely to cancel each other leaving a very small overall anisotropy. In fact, we have observed that the total magneto-anisotropy energy 
is only of order $0.1 k_{s}$. Our approach is purely based on thermodynamics and presents no information on the spin dynamics of the nanoparticle. It is very likely that the surface spins form a disordered glass-like layer, which slows down the reversal of magnetization.

\section{ACKNOWLEDGEMENTS}

This research is supported by the Department of Energy through the Laboratory Technology Research Program of OASCR and the Computational Materials Science Network of BES under Contract No. DE-AC05-00OR22725 with UT-Battelle LLC, and also by NSF DMR-0341874.

1 S. N. Khanna and S. Linderoth, Phys. Rev. Lett. 67, 742 (1991).

2 E. H. Frei, et. al., Phys. Rev. 106, 446 (1957).

3 R. H. Kodama and A. E. Berkowitz, Phys. Rev. B, 59, 6321 (1999).

4 R. H. Kodama, et. al., Phys. Rev. Lett. 77, 394 (1996).

5 R. H. Kodama, J. Mag. Mag. Mat. 200, 359 (1999).

6 Y. Miyatake, et. al. J. Phys. C 19, 2539 (1986).

7 D. Loison, et. al. Eur. Phys. J. B 41, 395 (2004).

8 C. Zhou, et. al. Phys. Rev. B 69, 144419 (2004).

9 F. Wang and D. P. Landau, Phys. Rev. Lett. 86, 2050 (2001); Phys. Rev. E 64, 056101, (2001); Comput.Phys. Commun. 147, 674 (2002).

10 C. Zhou, et. al. cond-mat/0509335.

11 J. Smith and H. P. J. Wijn, Ferrites, John Wiley \& Sons Publishers (1959). 